\title{
NAYATANI'S METRIC AND CONFORMAL TRANSFORMATIONS OF A KLEINIAN MANIFOLD
}

\author{
YASUHIRO YABUKI
}

(Communicated by Richard A. Wentworth)

\begin{abstract}
According to Schoen and Yau (1988), an extensive class of conformally flat manifolds is realized as Kleinian manifolds. Nayatani (1997) constructed a metric on a Kleinian manifold $M$ which is compatible with the canonical flat conformal structure. He showed that this metric $g_{N}$ has a large symmetry if $g_{N}$ is a complete metric. Under certain assumptions including the completeness of $g_{N}$, the isometry group of $\left(M, g_{N}\right)$ coincides with the conformal transformation group of $M$. In this paper, we show that $g_{N}$ may have a large symmetry even if $g_{N}$ is not complete. In particular, every conformal transformation is an isometry when $\left(M, g_{N}\right)$ corresponds to a geometrically finite Kleinian group.
\end{abstract}

\section{INTRODUCTION}

Let $M$ be a differentiable manifold of dimension $n \geq 3$ with a conformal structure $\mathcal{C}$ which is an equivalence class of metrics on $M$ conformal to each other. $(M, \mathcal{C})$ is said to be conformally flat if for some (hence for any) $g \in \mathcal{C}, g$ has an expression of the form

$$
g=\lambda(x) \sum_{k=1}^{n}\left(d x^{i}\right)^{2}
$$

for some coordinates and function $\lambda>0$ locally. Schoen and Yau [9] showed that there is a large class of conformally flat manifolds that can be realized as Kleinian manifolds. In particular, this class contains all compact conformally flat manifolds $(M, \mathcal{C})$ of dimension $n \geq 4$ that satisfy $R_{g}>0$ for some $g \in \mathcal{C}$, where $R_{g}$ is the scalar curvature of $g$. Here a Kleinian manifold is of the form $\Omega / \Gamma$ where $\Omega$ is a domain of $S^{n}$ and $\Gamma$ is a Kleinian group acting freely and properly discontinuously on $\Omega$ (see the details below).

We let $B^{n+1}$ be the Poincaré disk model of hyperbolic $n+1$ space with the Poincaré metric $g_{H}$. Each isometry of $\left(B^{n+1}, g_{H}\right)$ naturally extends to a diffeomorphism on the ideal boundary $S^{n}\left(=\partial B^{n+1}\right)$ as a conformal transformation with respect to the standard metric $g_{0}$. In this way, the isometry group of $B^{n+1}$ may

Received by the editors June 15, 2006 and, in revised form, November 24, 2006.

2000 Mathematics Subject Classification. Primary 53A30; Secondary 22E40.

Key words and phrases. Nayatani's metric, geometrically finite, conformally flat.

(C) 2007 American Mathematical Society 
be identified with the conformal transformation group of $S^{n}: \operatorname{Isom}\left(B^{n+1}, g_{H}\right) \cong$ $\operatorname{Conf}\left(S^{n}, g_{0}\right)$. Throughout this paper, we regard $B^{n+1}$ and $S^{n}$ as subsets of $\mathbb{R}^{n+1}$, that is,

$$
B^{n+1}=\left\{x \in \mathbb{R}^{n+1}|| x \mid<1\right\}, \quad S^{n}=\left\{x \in \mathbb{R}^{n+1}|| x \mid=1\right\} .
$$

Let $\Gamma$ be a Kleinian group, that is, an infinite discrete subgroup of the conformal transformation group $\operatorname{Conf}\left(S^{n}, g_{0}\right)$. The largest open subset of $S^{n}$ on which $\Gamma$ acts properly discontinuously is called the domain of discontinuity of $\Gamma$, denoted by $\Omega(\Gamma)$. The set $\Omega(\Gamma)$ is characterized as the complement $S^{n} \backslash \Lambda(\Gamma)$, where $\Lambda(\Gamma)$ is the set of accumulation points of $\Gamma x, x \in B^{n+1}$, in $\overline{B^{n+1}}\left(=B^{n+1} \cup S^{n}\right)$. Notice that $\Lambda(\Gamma)$, called the limit set of $\Gamma$, is independent of the choice of $x \in B^{n+1}$. Nayatani [6] constructed a metric on $\Omega(\Gamma)$ which is compatible with the canonical flat conformal structure induced by $g_{0}$ :

$$
g_{N}=\left(\int_{\Lambda(\Gamma)}\left(\frac{2}{|y-\xi|^{2}}\right)^{\delta(\Gamma)} d \mu_{\delta(\Gamma)}(\xi)\right)^{2 / \delta(\Gamma)} g_{0}, \quad y \in \Omega(\Gamma),
$$

where $\delta(\Gamma)$ denotes the critical exponent of $\Gamma$ and $\mu_{\delta(\Gamma)}$ is a Patterson-Sullivan measure of exponent $\delta(\Gamma)$ (see the definitions in Sect. 2).

Sullivan [11] proved the following theorem on the uniqueness of $\mu_{\delta(\Gamma)}$ for a large class of Kleinian groups.

Theorem 1.1 (Sullivan [11]). Suppose that $\Gamma$ is a geometrically finite Kleinian group. Then the critical exponent $\delta(\Gamma)$ equals the Hausdorff dimension $d(\Gamma)$ of the limit set $\Lambda(\Gamma)$. Moreover if $\Gamma$ is nonelementary, there exists, up to a constant multiple, one and only one Patterson-Sullivan measure of exponent $\delta(\Gamma)$.

We say that $\Gamma$ is convex cocompact if the quotient $C(\Lambda(\Gamma)) / \Gamma$ is compact, where $C(\Lambda(\Gamma))$ is the hyperbolic convex hull of $\Lambda(\Gamma)$ in $B^{n+1}$. Such a $\Gamma$ is characterized as a geometrically finite group without parabolic elements. It is known that if $\Gamma$ is nonelementary and convex cocompact, the unique Patterson-Sullivan measure $\mu_{\delta(\Gamma)}$ coincides (up to a constant multiple) with the $\delta(\Gamma)$-dimensional Hausdorff measure $\mathcal{H}^{\delta(\Gamma)}$ on the limit set $\Lambda(\Gamma)$ of $\Gamma$.

Nayatani's metric $g_{N}$ is $\Gamma$-invariant. Therefore it projects to a conformally flat metric on the quotient manifold $\Omega / \Gamma$ if $\Gamma$ acts freely and properly discontinuously on a domain $\Omega \subset \Omega(\Gamma)$. We denote this metric on $\Omega / \Gamma$ by the same symbol $g_{N}$. Nayatani's metric $g_{N}$, by construction, has remarkable properties in connection with Kleinian group theory. For instance, there is a close relation between the curvature of $g_{N}$ and the critical exponent $\delta(\Gamma)$ of $\Gamma$. Namely, if the limit set of $\Gamma$ contains at least two points, the scalar curvature of $g_{N}$ is positive (resp. zero, negative) if and only if $\delta(\Gamma)<($ resp. $=,>)(n-2) / 2\left(\right.$ see $\left[6\right.$, Theorem 3.3]). Besides this, $g_{N}$ has the following property, which gives the motivation of this paper.

Theorem 1.2 (Nayatani [6]). Let $M=\Omega / \Gamma$ be a Kleinian manifold with $\delta(\Gamma)>0$ and $g_{N}$ a Nayatani's metric. Suppose that $\mu_{\delta(\Gamma)}$ is, up to a constant multiple, the unique Patterson-Sullivan measure of exponent $\delta(\Gamma)$ and that $g_{N}$ is complete. Then the isometry group $\operatorname{Isom}\left(M, g_{N}\right)$ coincides with the conformal transformation group $\operatorname{Conf}\left(M, g_{N}\right)$.

This theorem says that $g_{N}$ has a large symmetry if $g_{N}$ is a complete metric. In particular, by Theorem 1.1, we have that $\operatorname{Conf}\left(M, g_{N}\right)=\operatorname{Isom}\left(M, g_{N}\right)$ for a 
Kleinian manifold $M$ which corresponds to a convex cocompact group $\Gamma$. The purpose of this paper is to show that $g_{N}$ may have a large symmetry even if $g_{N}$ is not complete. More precisely speaking,

Theorem 3.5. Let $M=\Omega / \Gamma$ be a Kleinian manifold with $\delta(\Gamma)>0$ and $g_{N}$ a Nayatani's metric on $M$. Suppose that $\Gamma$ is geometrically finite. Then the isometry group $\operatorname{Isom}\left(M, g_{N}\right)$ coincides with the conformal transformation group $\operatorname{Conf}\left(M, g_{N}\right)$.

Theorem 3.5 is not a direct consequence of Theorem 1.2. Indeed Nayatani's metric $g_{N}$ is not necessarily complete even if $\Gamma$ is geometrically finite. In fact, Maubon [5] gave the necessary and sufficient condition for $g_{N}$ to be complete/incomplete when $\Gamma$ is geometrically finite.

This paper is organized as follows. In Sect. 2, we review some facts on PattersonSullivan measures and Nayatani's metric. In Sect. 3, we shall prove Theorem 3.5, which is a result for geometrically finite groups. In Sect. 4 , we consider the case that Kleinian groups are not necessarily geometrically finite.

\section{Patterson-Sullivan measures and Nayatani's metric}

We start this section with the definition and fundamental properties of PattersonSullivan measures. More details may be found in [8] for example.

For a Kleinian group $\Gamma$, the critical exponent $\delta(\Gamma)$ is defined by

$$
\delta(\Gamma)=\inf \left\{\begin{array}{l|l|}
s>0 \mid \sum_{\gamma \in \Gamma} \exp \left\{-s d_{H}(x, \gamma y)\right\}<\infty
\end{array}\right\},
$$

where $x, y \in B^{n+1}$ and $d_{H}$ is the hyperbolic distance function on $B^{n+1}$. It is easy to see that $\delta(\Gamma)$ is independent of the choice of $x, y \in B^{n+1}$. It is known that $0 \leq \delta(\Gamma) \leq n$ (see [8, Theorem 1.6.1]). A Kleinian group $\Gamma$ is called nonelementary if $\Lambda(\Gamma)$ contains at least three points. If $\Gamma$ is nonelementary, $\delta(\Gamma)>0$ (see [8, Corollary 3.4.5]).

Definition 2.1. For a Kleinian group $\Gamma$ and $\rho>0$, we define a Patterson-Sullivan measure $\mu_{\rho}$ of exponent $\rho$ as a positive finite Borel measure on $S^{n}$ which satisfies the following conditions:

(i) $\mu_{\rho}$ is supported on $\Lambda(\Gamma)$.

(ii)

$$
\gamma^{*} \mu_{\rho}=\left|\gamma^{\prime}\right|^{\rho} \mu_{\rho}, \quad \gamma \in \Gamma,
$$

where $\left|\gamma^{\prime}\right|$ denotes the linear stretch factor of $\gamma$ defined by

$$
\gamma^{*} g_{0}=\left|\gamma^{\prime}\right|^{2} g_{0}
$$

The condition (2.1) implies that a Patterson-Sullivan measure of exponent $\rho$ locally behaves like the $\rho$-dimensional Hausdorff measure on $\Lambda(\Gamma)$. Notice that a Patterson-Sullivan measure $\mu_{\rho}$ is nothing but a measure $\sigma_{o}$, where $o$ is the origin of $B^{n+1}$ and $\left\{\sigma_{x}\right\}_{x \in B^{n+1}}$ is a $\Gamma$-invariant conformal density of dimension $\rho$ (see [8, Chapter 4]). Here a $\Gamma$-invariant conformal density of dimension $\rho$ is a family of positive finite Borel measures $\sigma_{x}, x \in B^{n+1}$, on $S^{n}$ that are supported on $\Lambda(\Gamma)$ and satisfy the following transformation laws:

$$
\begin{gathered}
\gamma^{*} \sigma_{x}=\sigma_{\gamma^{-1} x}, \quad x \in B^{n+1}, \gamma \in \Gamma, \\
\sigma_{x}=P(x, \cdot)^{\rho} \sigma_{o}, \quad x \in B^{n+1}, \gamma \in \Gamma,
\end{gathered}
$$


where $P$ is defined by

$$
P(x, y)=\frac{1-|x|^{2}}{|x-y|^{2}}, \quad x \in B^{n+1}, y \in S^{n} .
$$

Set $\sigma=\sigma_{x}$. Then by (2.2) and (2.3) we have

$$
\gamma^{*} \sigma=\left(\frac{P\left(\gamma^{-1} x, \cdot\right)}{P(x, \cdot)}\right)^{\rho} \sigma, \quad \gamma \in \Gamma
$$

We say that a measure $\sigma$ on $S^{n}$ is a Patterson-Sullivan measure of exponent $\rho$ with basepoint $x$ if $\sigma$ is a positive finite Borel measure supported on $\Lambda(\Gamma)$ that satisfies (2.5). Let $x \in B^{n+1}$ and $\alpha \in \operatorname{Conf}\left(S^{n}, g_{0}\right)$. We regard $\alpha$ as a diffeomorphism of $B^{n+1} \cup S^{n}$ through the identification $\operatorname{Isom}\left(B^{n+1}, g_{H}\right) \cong \operatorname{Conf}\left(S^{n}, g_{0}\right)$. Then the linear stretch factor of $\alpha$ with respect to $g_{0}$ is given by

$$
\begin{aligned}
\left|\alpha^{\prime}(y)\right| & =\frac{P(x, y)}{P(\alpha x, \alpha y)} \\
& =P\left(\alpha^{-1} o, y\right),
\end{aligned}
$$

where $x \in B^{n+1}, y \in S^{n}$ and $o$ is the origin of $B^{n+1}$. Patterson-Sullivan measures were introduced by Patterson [7] and Sullivan [10]. They constructed such a measure in the case of exponent $\delta(\Gamma)$. It is known that if there exists a PattersonSullivan measure $\mu_{\rho}$ of exponent $\rho$, then $\rho \geq \delta(\Gamma)$ (see [8, Corollary 4.5.3]). However, complete answers are not known to the existence and uniqueness problems of Patterson-Sullivan measures $\mu_{\rho}$ of exponent $\rho>\delta(\Gamma)$.

Let $\Gamma$ be a Kleinian group with $\delta(\Gamma)>0$. Nayatani's metric $(1.1)$ on $\Omega(\Gamma)$ is naturally extended to a metric on $B^{n+1}$ as the following:

$$
\begin{aligned}
g_{N} & =\left(\int_{\Lambda(\Gamma)}\left(\frac{2}{|x-\xi|^{2}}\right)^{\delta(\Gamma)} d \mu_{\delta(\Gamma)}(\xi)\right)^{2 / \delta(\Gamma)} g_{E} \\
& =\left(\int_{\Lambda(\Gamma)}\left(\frac{1-|x|^{2}}{|x-\xi|^{2}}\right)^{\delta(\Gamma)} d \mu_{\delta(\Gamma)}(\xi)\right)^{2 / \delta(\Gamma)} g_{H},
\end{aligned}
$$

where $x \in B^{n+1}$ and $g_{E}$ denotes the Euclidean metric (see [3]). This metric $g_{N}$ is also $\Gamma$-invariant on $B^{n+1}$. Therefore if $\Gamma$ acts freely and properly discontinuously on $B^{n+1}, g_{N}$ projects to a metric, denoted by the same symbol $g_{N}$, on the quotient manifold $B^{n+1} / \Gamma$. Since $\Gamma$ acts on $B^{n+1}$ as isometries of $\left(B^{n+1}, g_{H}\right), B^{n+1} / \Gamma$ is a hyperbolic manifold.

Throughout this paper, we assume that a Kleinian manifold $M$ is the quotient of a connected open subset $\Omega \subset \Omega(\Gamma)$ by $\Gamma, M=\Omega / \Gamma$, with the canonical flat conformal structure $\mathcal{C}_{0}$. Here $\Omega$ is $\Gamma$-invariant and $\Gamma$ acts freely on $\Omega$. We also assume $\operatorname{dim} M \geq 3$.

Our major concern in this paper is with the isometry group of a Kleinian manifold $\left(M, g_{N}\right)$. For the conformal transformation group of $\left(M, \mathcal{C}_{0}\right)$, we know the following.

Proposition 2.2 (Nayatani $[6]$ ). Let $M=\Omega / \Gamma$ be a Kleinian manifold. Then the conformal transformation group $\operatorname{Conf}(M)$ of $M$ is isomorphic to $(N(\Gamma) \cap$ $\left.\operatorname{Conf}\left(\Omega, g_{0}\right)\right) / \Gamma$, where $N(\Gamma)$ denotes the normalizer of $\Gamma$ in $\operatorname{Conf}\left(S^{n}, g_{0}\right)$. 
We shall now sketch a proof of Proposition 2.2. For $\alpha \in N(\Gamma) \cap \operatorname{Conf}\left(\Omega, g_{0}\right)$, we define $\underline{\alpha} \in \operatorname{Conf}(M)$ as follows:

$$
\underline{\alpha}(\Gamma y)=\Gamma \alpha y, \quad y \in \Omega,
$$

where for $x \in \Omega, \Gamma x$ is regarded as a point in $M$. Note that $\underline{\alpha}$ is well defined. Letting $f(\alpha)=\underline{\alpha}$, we obtain a homomorphism $f: N(\Gamma) \cap \operatorname{Conf}\left(\Omega, g_{0}\right) \longrightarrow \operatorname{Conf}(M)$. We can verify that $f$ is surjective and the kernel of $f$ coincides with $\Gamma$ (see $[6$, Lemma 4.1]). Therefore $\operatorname{Conf}(M)$ is isomorphic to $\left(N(\Gamma) \cap \operatorname{Conf}\left(\Omega, g_{0}\right)\right) / \Gamma$.

\section{Geometrichlly finite case}

In this section, we give a proof of Theorem 3.5 which is a result for geometrically finite Kleinian groups. A Kleinian group $\Gamma$ is called geometrically finite if it has some convex fundamental polyhedron in $B^{n+1}$ with finitely many faces. Geometrical finiteness is one of the most important notions in Kleinian group theory, particularly from the viewpoint of hyperbolic geometry. In the context of hyperbolic 3-manifolds, several equivalent definitions of geometrical finiteness are given by Beardon and Maskit [1], Marden [4], and Thurston [12]. Moreover Bowditch [2] gave equivalent definitions of geometrical finiteness in general dimension. The following is one of them:

(GF) For some $\epsilon>0$, the $\epsilon$-thick part of the convex core $C_{\Gamma} \subset B^{n+1} / \Gamma$ is compact. Here the convex core is defined by $C_{\Gamma}=C(\Lambda(\Gamma)) / \Gamma$, where $C(\Lambda(\Gamma))$ is the hyperbolic convex hull of $\Lambda(\Gamma)$ in $B^{n+1}$.

We shall now show the following lemma, which is obtained by combining [6, Lemma 4.2] with [6, Lemma 4.3].

Lemma 3.1. Let $\Gamma$ be a Kleinian group with $\delta(\Gamma)>0, g_{N}$ a Nayatani's metric, and $\alpha$ an element of $N(\Gamma)$. Suppose that $\mu_{\delta(\Gamma)}$ is, up to a constant multiple, the unique Patterson-Sullivan measure of exponent $\delta(\Gamma)$. Then $\alpha$ is a homothety with respect to $g_{N}$; that is,

$$
\alpha^{*} g_{N}=c g_{N}
$$

for some constant $c>0$. We also have, for this constant $c$,

$$
\alpha^{*} \mu_{\delta(\Gamma)}=c^{\delta(\Gamma) / 2}\left|\alpha^{\prime}\right|^{\delta(\Gamma)} \mu_{\delta(\Gamma)} .
$$

Proof. It is known that $\alpha^{*} \mu_{\delta(\Gamma)}$ is a Patterson-Sullivan measure of exponent $\delta(\Gamma)$ with basepoint $\alpha^{-1} o$ (see [6, Lemma 4.2]). It follows from (2.3), (2.7) and the uniqueness of $\mu_{\delta(\Gamma)}$ that

$$
\alpha^{*} \mu_{\delta(\Gamma)}=\tilde{c}\left|\alpha^{\prime}\right|^{\delta(\Gamma)} \mu_{\delta(\Gamma)}
$$

for some constant $\tilde{c}>0$. We now recall the following formula:

$$
|\gamma y-\gamma \xi|^{2}=\left|\gamma^{\prime}(y)\right|\left|\gamma^{\prime}(\xi)\right||y-\xi|^{2}, \quad y, \xi \in S^{n}, \gamma \in \operatorname{Conf}\left(S^{n}, g_{0}\right) \text {. }
$$


Using these, we compute

$$
\begin{aligned}
\alpha^{*} g_{N} & =\left(\int_{\Lambda(\Gamma)}\left(\frac{2}{|\alpha y-\xi|^{2}}\right)^{\delta(\Gamma)} d \mu_{\delta(\Gamma)}(\xi)\right)^{2 / \delta(\Gamma)} \alpha^{*} g_{0} \\
& =\left(\int_{\Lambda(\Gamma)}\left(\frac{2}{|\alpha y-\alpha \xi|^{2}}\right)^{\delta(\Gamma)} d\left(\alpha^{*} \mu_{\delta(\Gamma)}\right)(\xi)\right)^{2 / \delta(\Gamma)}\left|\alpha^{\prime}(y)\right|^{2} g_{0} \\
& =\left(\int_{\Lambda(\Gamma)}\left(\frac{2}{\left|\alpha^{\prime}(y)\right|\left|\alpha^{\prime}(\xi)\right||y-\xi|^{2}}\right)^{\delta(\Gamma)} \tilde{c}\left|\alpha^{\prime}(\xi)\right|^{\delta(\Gamma)} d \mu_{\delta(\Gamma)}(\xi)\right)^{2 / \delta(\Gamma)}\left|\alpha^{\prime}(y)\right|^{2} g_{0} \\
& =\tilde{c}^{2 / \delta(\Gamma)} g_{N}
\end{aligned}
$$

on $\Omega(\Gamma)$. When we regard $g_{N}$ as a metric on $B^{n+1}$, we also have

$$
\begin{aligned}
\alpha^{*} g_{N} & =\left(\int_{\Lambda(\Gamma)} P(\alpha x, \xi)^{\delta(\Gamma)} d \mu_{\delta(\Gamma)}(\xi)\right)^{2 / \delta(\Gamma)} \alpha^{*} g_{H} \\
& =\left(\int_{\Lambda(\Gamma)} P(\alpha x, \alpha \xi)^{\delta(\Gamma)} d\left(\alpha^{*} \mu_{\delta(\Gamma)}\right)(\xi)\right)^{2 / \delta(\Gamma)} g_{H} \\
& =\left(\int_{\Lambda(\Gamma)}\left(\left|\alpha^{\prime}(\xi)\right|^{-1} P(x, \xi)\right)^{\delta(\Gamma)} \tilde{c}\left|\alpha^{\prime}(\xi)\right|^{\delta(\Gamma)} d \mu_{\delta(\Gamma)}(\xi)\right)^{2 / \delta(\Gamma)} g_{H} \\
& =\tilde{c}^{2 / \delta(\Gamma)} g_{N} .
\end{aligned}
$$

by (2.6) and (2.8). Putting $c=\tilde{c}^{2 / \delta(\Gamma)}$ completes the proof.

We now prove

Proposition 3.2. Let $M=\Omega / \Gamma$ be a Kleinian manifold with $\delta(\Gamma)>0, g_{N} a$ Nayatani's metric, and $\alpha \in N(\Gamma) \cap \operatorname{Conf}\left(\Omega, g_{0}\right)$. Suppose that $\mu_{\delta(\Gamma)}$ is, up to a constant multiple, the unique Patterson-Sullivan measure of exponent $\delta(\Gamma)$ and that $\alpha$ has a fixed point in $\Omega$ or $\underline{\alpha}$ has a fixed point in $M$ (see the definition of $\underline{\alpha}$ in Sect. 2). Then $\alpha$ (hence $\underline{\alpha})$ is an isometry with respect to $g_{N}$.

Proof. It follows from Lemma 3.1 that $\alpha$ is a homothety. Therefore $\alpha^{*} g_{N}=c g_{N}$ (and $\underline{\alpha}^{*} g_{N}=c g_{N}$ ) for some constant $c>0$. Suppose $c \neq 1$. Considering $\alpha^{-1}$ if necessary, we may assume $c<1$. If $\alpha$ has a fixed point in $\Omega$, then $\underline{\alpha}$ also has a fixed point in $M$. Hence we may assume that $\underline{\alpha}$ has a fixed point $p$ in $\bar{M}$. Then any loop in $M$ is mapped into a simply connected neighborhood of $p$ by $\underline{\alpha}^{k}$ for a sufficiently large integer $k$. So $M$ is simply connected (actually contractible). Recalling that $\Gamma$ is the image of $\pi_{1}(M)$ by the holonomy representation, one sees that $\Gamma$ is trivial. This contradicts $\delta(\Gamma)>0$. This completes the proof.

Remark 3.3. The proof of Proposition 3.2 slightly simplifies that of Theorem 1.2, because we can find a fixed point of a contracting homothety if $\left(M, g_{N}\right)$ is complete. In fact $\left\{\underline{\alpha}^{k}(p)\right\}_{k=1}^{\infty}$, for $p \in M$ and a contracting homothety $\underline{\alpha}$, is a Cauchy sequence and hence converges to a point $q \in M$. This point $q$ is fixed by $\alpha$. 
Let $\Gamma$ be a Kleinian group. For $\alpha \in N(\Gamma)$, we extend the domain of $\underline{\alpha}$. Namely we define a homeomorphism $\underline{\alpha}:\left[B^{n+1} \cup \Omega(\Gamma)\right] / \Gamma \longrightarrow\left[B^{n+1} \cup \Omega(\Gamma)\right] / \Gamma$ as the following:

$$
\underline{\alpha}(\Gamma x)=\Gamma \alpha x, \quad x \in B^{n+1} \cup \Omega(\Gamma) .
$$

Proposition 3.4. Let $\Gamma$ be a finitely generated Kleinian group with the unique Patterson-Sullivan measure of exponent $\delta(\Gamma)>0$, and let $g_{N}$ be the Nayatani's metric. Suppose that $\alpha$ has a fixed point in $B^{n+1}$ or $\underline{\alpha}$ has a fixed point in $B^{n+1} / \Gamma$. Then $\alpha$ is an isometry with respect to $g_{N}$.

Proof. By the Selberg lemma, there exists a normal torsion-free subgroup $\Gamma^{\prime} \subset \Gamma$ of finite index. Such a $\Gamma^{\prime}$ satisfies $\delta\left(\Gamma^{\prime}\right)=\delta(\Gamma), \Lambda\left(\Gamma^{\prime}\right)=\Lambda(\Gamma), N\left(\Gamma^{\prime}\right)=N(\Gamma)$ and a Patterson-Sullivan measure $\mu_{\delta\left(\Gamma^{\prime}\right)}$ of $\Gamma^{\prime}$ coincides with $\mu_{\delta(\Gamma)}$. The argument in the proof of Proposition 3.2 implies that $\Gamma^{\prime}$ is trivial and hence that $\Gamma$ must be a finite group, a contradiction. This completes the proof.

By Proposition 3.4 and (GF), we have the following.

Theorem 3.5. Let $M=\Omega / \Gamma$ be a Kleinian manifold with $\delta(\Gamma)>0$, and let $g_{N}$ be a Nayatani's metric on $M$. Suppose that $\Gamma$ is geometrically finite. Then the isometry group $\operatorname{Isom}\left(M, g_{N}\right)$ coincides with the conformal transformation group $\operatorname{Conf}\left(M, g_{N}\right)$.

Proof. By Proposition 2.2, it is sufficient to show that $\alpha^{*} g_{N}=g_{N}$ for any $\alpha \in$ $N(\Gamma) \cap \operatorname{Conf}\left(\Omega, g_{0}\right)$. It follows from Lemma 3.1 that $\alpha$ is a homothety; that is, $\alpha^{*} g_{N}=c g_{N}$ for some constant $c>0$. Suppose $c \neq 1$. We may assume $c<1$. The $\epsilon$-thick part of the convex core $C_{\Gamma}$, by $(\mathrm{GF})$, is compact and is preserved by the action of $\alpha$ because $\alpha$ acts isometrically on $C_{\Gamma}$ in the hyperbolic sense. Here we used the fact that $\alpha$ leaves $\Lambda(\Gamma)$ invariant. Therefore for a point $x$ in the $\epsilon$-thick part of $C_{\Gamma},\left\{\underline{\alpha}^{k}(x)\right\}_{k=1}^{\infty}$, taking a subsequence if necessary, converges to some point $\tilde{x} \in C_{\Gamma} \subset B^{n+1} / \Gamma$. Then $\tilde{x}$ is a fixed point of $\underline{\alpha}$. In fact, since $\underline{\alpha}$ is a homothety, the sequence $\left\{\underline{\alpha}^{k}(x)\right\}_{k=1}^{\infty}$ itself converges, and the limit must be fixed by $\underline{\alpha}$. Since geometrically finite Kleinian groups are finitely generated, Proposition 3.4 completes the proof.

\section{General CASe}

From what we have seen so far, it is plausible that $\operatorname{Isom}\left(M, g_{N}\right)=\operatorname{Conf}\left(M, g_{N}\right)$ holds for every Kleinian manifold $\left(M, g_{N}\right)$ corresponding to a Kleinian group $\Gamma$ with the unique Patterson-Sullivan measure of exponent $\delta(\Gamma)$. In this section, we show a result which may be considered as a partial evidence to this statement. First, we show the following.

Theorem 4.1. Let $M=\Omega / \Gamma$ be a Kleinian manifold with $\delta(\Gamma)>0$, and let $g_{N}$ be a Nayatani's metric. Suppose that there is no Patterson-Sullivan measure $\mu_{\rho}$ of exponent $\rho \neq \delta(\Gamma)$ and that $\mu_{\delta(\Gamma)}$ is unique up to a constant multiple. Then the isometry group $\operatorname{Isom}\left(M, g_{N}\right)$ coincides with the conformal transformation group $\operatorname{Conf}\left(M, g_{N}\right)$.

Proof. By Proposition 2.2, it is sufficient to show that $\alpha^{*} g_{N}=g_{N}$ for any $\alpha \in$ $N(\Gamma) \cap \operatorname{Conf}\left(\Omega, g_{0}\right)$. It follows from Lemma 3.1 that $\alpha$ is a homothety; that is, $\alpha^{*} g_{N}=c g_{N}$ for some constant $c>0$. Suppose $c \neq 1$. Considering $\alpha^{-1}$ if necessary, we may assume $c<1$. By Proposition 3.2, we may also assume that neither $\alpha$ nor 
$\underline{\alpha}$ have a fixed point in $\Omega$ or $M$. Then $\langle\alpha\rangle$ (resp. $\langle\underline{\alpha}\rangle)$ acts properly discontinuously on $\Omega$ (resp. $M$ ). If not, since $\alpha$ (hence $\underline{\alpha}$ ) is a contracting homothety, $\alpha$ or $\underline{\alpha}$ has a fixed point in $\Omega$ or $M$. In fact, for a compact subset $K$ of $\Omega$ such that

$$
\sharp\left\{k \in \mathbb{Z} \mid \alpha^{k} K \cap K \neq \emptyset\right\}=\infty,
$$

$\left\{\alpha^{k}(y)\right\}_{k=1}^{\infty}, y \in K$, has an accumulation point $y^{\prime} \in K$ and this point $y^{\prime}$ is fixed by $\alpha$. We now let $\tilde{\Gamma}=\langle\Gamma, \alpha\rangle$. Suppose $\tilde{\Gamma}$ is discrete. Then we have $\delta(\tilde{\Gamma})=\delta(\Gamma)$ as follows. First, we recall that the action of $N(\Gamma)$ leaves $\Lambda(\Gamma)$ invariant and if $\delta(\Gamma)>$ 0 , then $\Lambda(\Gamma)$ is the smallest closed subset of $S^{n}$ that is $\Gamma$-invariant. This means that $\Lambda(\tilde{\Gamma})=\Lambda(\Gamma)$. Therefore each Patterson-Sullivan measure $\tilde{\mu}_{\delta(\tilde{\Gamma})}$ of $\tilde{\Gamma}$ is also a Patterson-Sullivan measure of $\Gamma$. By the hypothesis that the exponent of PattersonSullivan measures is unique, we have $\delta(\tilde{\Gamma})=\delta(\Gamma)$. Moreover it follows from the uniqueness of $\mu_{\delta(\Gamma)}$ that $\tilde{\mu}_{\delta(\tilde{\Gamma})}$ coincides with $\mu_{\delta(\Gamma)}$ up to a constant multiple. The transformation law (2.1) of Patterson-Sullivan measures, with (3.2), implies that $c=1$. This is a contradiction.

We next consider the case that $\tilde{\Gamma}$ is not discrete. Let $\left\{\tilde{\gamma}_{i}\right\}_{i=1}^{\infty} \subset \tilde{\Gamma}$ be a sequence that converges to the identity. Since $\alpha$ is an element of $N(\Gamma)$, each $\tilde{\gamma}_{i}$ is represented by the form $\gamma_{i} \alpha^{k_{i}}$, where $\gamma_{i} \in \Gamma$ and $k_{i}$ is some integer. If $\left\{k_{i}\right\}_{i=1}^{\infty}$ is bounded, then there exist some $k \in \mathbb{Z}$ and subsequence $\left\{\gamma_{i(j)}\right\}_{j=1}^{\infty}$ such that

$$
\lim _{j \rightarrow \infty} \gamma_{i(j)}=\alpha^{k}
$$

This means that $\alpha^{k}$ is an element of $\Gamma$, and hence $c=1$. It remains to consider the case that $\left\{k_{i}\right\}_{i=1}^{\infty}$ is not bounded. Notice that each $\tilde{\gamma}_{i}=\gamma_{i} \alpha^{k_{i}}$ equals $\underline{\alpha}^{k_{i}}$ as an element of $\operatorname{Conf}\left(M, g_{N}\right)$. However $\langle\underline{\alpha}\rangle$ acts properly discontinuously on $\Omega / \Gamma$. This contradicts the assumption that $\tilde{\gamma}_{i}$ converges to the identity. This completes the proof.

Remark 4.2. Actually, we can show that Kleinian groups satisfying the conditions in Theorem 4.1 are convex cocompact. If we can show that $\delta(\Gamma)=\delta(\tilde{\Gamma})$ (see the proof of Theorem 4.1), we can drop the assumption in Theorem 4.1 that there is no Patterson-Sullivan measure of exponent $\rho>\delta(\Gamma)$.

Next we recall the classification of elements of $\operatorname{Conf}\left(S^{n}, g_{0}\right)$ according to their dynamics on $B^{n+1} \cup S^{n}$. A transformation $\gamma \in \operatorname{Conf}\left(S^{n}, g_{0}\right)$ is called elliptic if $\gamma$ has fixed points in $B^{n+1}$, loxodromic if $\gamma$ is not elliptic and has exactly two fixed points in $S^{n}$, and parabolic otherwise. If $\gamma$ is parabolic, $\gamma$ has exactly one fixed point in $S^{n}$. Let $\Gamma$ be a Kleinian group with $\delta(\Gamma)>0, g_{N}$ a Nayatani's metric on $\Omega(\Gamma)$ and $\alpha \in N(\Gamma)$. We now suppose that $\mu_{\delta(\Gamma)}$ is, up to a constant multiple, the unique Patterson-Sullivan measure of exponent $\delta(\Gamma)$. It is not difficult to see that for $\alpha$ elliptic, $\alpha$ is an isometry of $\left(\Omega(\Gamma), g_{N}\right)$ (see Proposition 3.4 in the case $\Gamma$ is finitely generated). Moreover we can show the following.

Proposition 4.3. Let $\Gamma$ be a Kleinian group with $\delta=\delta(\Gamma)>0, g_{N}$ a Nayatani's metric on $\Omega(\Gamma)$, and $\alpha \in N(\Gamma)$. Suppose that $\mu$ is, up to a constant multiple, the unique Patterson-Sullivan measure of exponent $\delta(\Gamma)$ and that $\alpha$ is a parabolic element. Then $\alpha$ is an isometry of $\left(\Omega(\Gamma), g_{N}\right)$ and hence if $M=\Omega / \Gamma$ is a Kleinian manifold and $\alpha \in N(\Gamma) \cap \operatorname{Conf}\left(\Omega, g_{0}\right), \underline{\alpha}$ also acts isometrically on $\left(M, g_{N}\right)$. 
Proof. By Lemma 3.1, $\alpha$ is a homothety; that is, $\alpha^{*} g_{N}=c g_{N}$ for some constant $c>0$. Suppose $c \neq 1$. We may assume $c<1$. Since $\left(\alpha^{k}\right)^{*} g_{N}=c^{k} g_{N}$ for $k \in \mathbb{Z}$, by the formula (2.8), we have the following equation:

$$
\left(\int_{\Lambda(\Gamma)}\left(\frac{1}{\left|\alpha^{k} x-\xi\right|^{2}}\right)^{\delta} d \mu(\xi)\right)^{2 / \delta}=\frac{c^{k}}{\left(1-\left|\alpha^{k} x\right|^{2}\right)^{2}}\left(\int_{\Lambda(\Gamma)}\left(\frac{1-|x|^{2}}{|x-\xi|^{2}}\right)^{\delta} d \mu(\xi)\right)^{2 / \delta}
$$

where $x \in B^{n+1}$. We now compute the order of $\left(1-\left|\alpha^{k} x\right|^{2}\right)^{2}$ as $k \rightarrow \infty$. To do this, it is convenient to work with the upper half-space model $H^{n+1}=\left\{z \in \mathbb{R}^{n+1} \mid\right.$ $\left.z_{n+1}>0\right\}$ of the hyperbolic space. The identification $J: H^{n+1} \rightarrow B^{n+1}$ is given by the composition of the following two maps:

(i) $H^{n+1} \rightarrow\left\{y_{n+1}<\frac{1}{2}\right\} ; z \mapsto y=z+\left(\frac{1}{2}-2 z_{n+1}\right) e_{n+1}$,

(ii) $\left\{y_{n+1}<\frac{1}{2}\right\} \rightarrow B^{n+1} ; y \mapsto x=e_{n+1}+\left(y-e_{n+1}\right)\left|y-e_{n+1}\right|^{-2}$,

where $e_{n+1}$ denotes the $(n+1)$-th element of the standard basis of $\mathbb{R}^{n+1}$. By direct calculation, we have

$$
1-|x|^{2}=\frac{2 z_{n+1}}{\left|[z]_{n}\right|^{2}+\left(\frac{1}{2}+z_{n+1}\right)^{2}},
$$

where $x=J(z)$ and $[z]_{n}=\left(z_{1}, \ldots, z_{n}\right) \in \mathbb{R}^{n}$. We may assume $\alpha\left(e_{n+1}\right)=e_{n+1}$ in the Poincaré model $B^{n+1}$ for considering $\left(1-\left|\alpha^{k} x\right|^{2}\right)^{2}$. Because each parabolic element has a decomposition of the form $P^{-1} \alpha^{\prime} P$, where $P$ is an orthogonal transformation of $\mathbb{R}^{n+1}$ and $\alpha^{\prime}$ is a parabolic element of $\operatorname{Conf}\left(S^{n}, g_{0}\right)$ that fixes $e_{n+1}$. Correspondingly $\alpha(\infty)=\infty$ in the upper half-space model $H^{n+1}$. Then $\alpha$ has the following expression in $H^{n+1}$ :

$$
\alpha(z)=\left[\begin{array}{cc}
T & 0 \\
0 & 1
\end{array}\right] z+\left[\begin{array}{l}
a \\
0
\end{array}\right]
$$

where $T$ is an $n \times n$ orthogonal matrix and $a \in \mathbb{R}^{n} \backslash\{o\}$ (see [8, p. 13], for example). Taking $x$ as $\alpha^{k} o$ in the formula (4.2), since we have $J^{-1}(o)=\frac{1}{2} e_{n+1}$,

$$
1-\left|\alpha^{k} o\right|^{2} \geq \frac{1}{|a|^{2} k^{2}+1}=O\left(k^{-2}\right) .
$$

Therefore the right-hand side of (4.1) tends to 0 as $k \rightarrow \infty$. On the other hand, the left-hand side of (4.1) is larger than $\frac{\mu(\Lambda(\Gamma))}{16}>0$ because $\left|\alpha^{k} x-\xi\right|<2$ for any $\xi \in \Lambda(\Gamma)$, a contradiction. Thus $c$ must be 1 , and this completes the proof.

Remark 4.4. For $\alpha \in N(\Gamma)$ loxodromic, we cannot use the same argument as above, because, in this case, $\left(1-\left|\alpha^{k} x\right|^{2}\right)^{2}$ tends to 0 exponentially for any $x \in B^{n+1}$. In fact the action of $\alpha$ on $\overline{B^{n+1}}$ has a fixed point at $e_{n+1}$, taking conjugates by some orthogonal transformation of $\mathbb{R}^{n+1}$ if necessary. Accordingly, in $H^{n+1}, \alpha$ is represented by

$$
\alpha(z)=\lambda\left[\begin{array}{ll}
T & 0 \\
0 & 1
\end{array}\right]\left(z-\left[\begin{array}{l}
a \\
0
\end{array}\right]\right)
$$

where $\lambda \in \mathbb{R}_{>0} \backslash\{1\}, T$ is an $n \times n$ orthogonal matrix and $a \in \mathbb{R}^{n}$. This, with (4.2), means that $\left(1-\left|\alpha^{k} x\right|^{2}\right)^{2} \rightarrow 0$ exponentially. If we can show, under the same conditions as Proposition 4.3, that every loxodromic element of $N(\Gamma)$ acts isometrically on $\left(\Omega(\Gamma), g_{N}\right)$, we can remove the assumption in Theorem 4.1 that there is no Patterson-Sullivan measure of exponent $\rho>\delta(\Gamma)$. 


\section{ACKNOWLEDGMENTS}

The author would like to thank Professor Hiroyasu Izeki for useful advice and discussions. He also thanks Professor Katsuhiko Matsuzaki for some comments on the earlier version of this paper.

\section{REFERENCES}

1. A. Beardon and B. Maskit, Limit sets of Kleinian groups and finite sided fundamental polyhedra, Acta Math. 132 (1974), 1-12. MR0333164 (48:11489)

2. B. H. Bowditch, Geometrical finiteness for hyperbolic groups, J. Funct. Anal. 113 (1993), 245-317. MR1218098 (94e:57016)

3. H. Izeki and S. Nayatani, Canonical metric on the domain of discontinuity of a Kleinian group, Séminaire de théorie spectrale et géométrie GRENOBLE 16 (1998), 9-32. MR1666506 (2000a:53018)

4. A. Marden, The geometry of finitely generated Kleinian groups, Ann. of Math. (2) 99 (1974), 383-462. MR0349992 (50:2485)

5. J. Maubon, Geometrically finite Kleinian groups: The completeness of Nayatani's metric, C. R. Acad. Sci. Paris, 325 (1997), 1065-1070. MR1614003 (99a:30041)

6. S. Nayatani, Patterson-Sullivan measure and conformally flat metrics, Math. Z. 225 (1997), 115-131. MR1451336 (98g:53072)

7. S. J. Patterson, The limit set of a Fuchsian group, Acta Math. 136 (1976), 241-273. MR0450547 (56:8841)

8. P. J. Nicholls, The ergodic theory of discrete groups, LMS Lect. Notes Ser. 143, Cambridge University Press, Cambridge, 1989. MR1041575 (91i:58104)

9. R. Schoen and S. T. Yau, Conformally flat manifolds, Kleinian groups and scaler curvature, Invent. Math. 92, (1988) 47-71. MR931204 (89c:58139)

10. D. Sullivan, The density at infinity of a discrete group of hyperbolic motions, I. H. E. S. Publ. Math. 50 (1979), 171-202. MR556586 (81b:58031)

11. D. Sullivan, Entropy, Hausdorff measures old and new, and limit sets of geometrically finite Kleinian groups, Acta Math. 153 (1984), 259-277. MR766265 (86c:58093)

12. W. P. Thurston, The Geometry and Topology of 3-manifolds, notes, Princeton Univ. Math. Department, 1979.

Mathematical Institute, Tohoku University, 980-8578 Sendai, Japan

E-mail address: sa3m30@math.tohoku.ac.jp 